\title{
Slow convergence of graphs under mean curvature flow
}

\author{
KASHIF RASUL
}

\begin{abstract}
In this paper, we study the mean curvature flow of entire graphs in Euclidean space. Ecker and Huisken in [1] have shown that given some initial growth condition at infinity and bounded initial gradient, such graphs, when rescaled, become self-similar under this evolution. Furthermore the convergence is exponentially fast in time. Here we consider a weaker condition at infinity, and show that under mean curvature flow such a condition is preserved for the height of the graph during the extent of the evolution. Our main result then states that under this alternative condition at infinity and bounded gradient, the rescaled graphs also become self-similar, converging however at a slower (polynomial in time) rate.
\end{abstract}

\section{Introduction}

We denote a smooth family of embeddings from an open subset $M^{n}$ of $\mathbb{R}^{n}$ by $F_{t}=F(\cdot, t): M^{n} \rightarrow \mathbb{R}^{n+1}$ with $F_{t}\left(M^{n}\right)=M_{t}$ for $t>0$. We say that this family of hypersurfaces moves by mean curvature flow if

$$
\begin{aligned}
\frac{\partial F}{\partial t}(p, t) & =\vec{H}(F(p, t)), \\
F(p, 0) & =F_{0}(p),
\end{aligned}
$$

for $p \in M^{n}$ and $t \in I$. Here $\vec{H}(F(p, t))$ is the mean curvature vector of the hypersurface $M_{t}$ at $F(p, t)$ defined as:

$$
\vec{H}:=-H \vec{\nu},
$$

where $H$ is the mean curvature and $\vec{\nu}$ the outward unit normal to the hypersurface at the point $F(p, t)$. In the case when $M_{t}$ are entire graphs, i.e., $M_{t}=\operatorname{graph} w(\cdot, t)$, where $w: \mathbb{R}^{n} \times(0, \infty) \rightarrow \mathbb{R}$ is smooth, mean curvature flow (1.1) is equivalent, modulo tangential diffeomorphisms in $\mathbb{R}^{n}$, to the 
following quasilinear equation:

$$
\begin{aligned}
\frac{\partial w}{\partial t} & =\sqrt{1+|D w|^{2}} \operatorname{div}\left(\frac{D w}{\sqrt{1+|D w|^{2}}}\right), \\
w(\cdot, 0) & =w_{0}(\cdot) .
\end{aligned}
$$

Rather than studying this equation from a PDE point of view, it is more helpful to look at certain natural geometric quantities like the height of $M_{t}$ with respect to the hyperplane $\mathbb{R}^{n} \times 0$ defined by:

$$
u(p, t)=\left\langle x(p, t), \overrightarrow{\mathrm{e}}_{n+1}\right\rangle,
$$

and its evolution. One can also similarly look at the gradient function:

$$
v=\frac{1}{\vec{\nu} \cdot \mathrm{e}_{n+1}}=\sqrt{1+|D w|^{2}}
$$

and derive estimates for the curvature of the graph. This yields by the work of Ecker and Huisken in [1], the longtime existence of a solution to (1.2) for initial data with bounded gradient.

Further they have shown that under certain conditions on the initial hypersurface near infinity, the solution of mean curvature flow becomes asymptotically self-similar, a self-similar solution being one whose graph over $\mathbb{R}^{n}$ moves only by homothety. Modulo scaling this says that $M_{t}=\sqrt{t} M_{1}$ for $t>0$. Such solutions are given by the solution of the following elliptic equation:

$$
H+\frac{1}{2} F \cdot \vec{\nu}=0
$$

The PDE (1.3) arises naturally when we consider solutions "coming out of a cone" in the sense that for initial data which is a cone: $w_{0}(\lambda p)=\lambda w_{0}(p)$ for some $\lambda>0$, the solution is of the form $w(p, t)=\sqrt{t} w(p / \sqrt{t}, 1)$. Stavrou in [3] has shown that in fact one can replace the asymptotic condition at infinity of Ecker and Huisken by asking that the initial surface have a unique tangent cone at infinity to obtain solutions to (1.2) which converge uniformly to a self-similar solution as $t \rightarrow \infty$. He shows that this is the weakest possible condition in view of a counter example, however he does not obtain a rate of convergence.

In general, graphs will move out to infinity as $t$ approaches infinity with height and curvature proportional to $1 / \sqrt{t}$, and so in order to study the global shape of the solutions, one needs to rescale the surfaces back in such a 
way that they do not diverge off, but still retain a bound on their curvatures. As in [2], we consider

$$
\tilde{F}(s)=\frac{F(t)}{\sqrt{2 t+1}},
$$

where the new time variable is given by

$$
s=\frac{1}{2} \log (2 t+1),
$$

for $0 \leq s<\infty$. The rescaled mean curvature flow then becomes

$$
\frac{\partial \tilde{F}}{\partial s}=\overrightarrow{\tilde{H}}-\tilde{F}
$$

with the same initial condition

$$
\tilde{F}(\cdot, 0)=F(\cdot, 0) .
$$

Ecker and Huisken have then shown that if the initial graph has bounded gradient

$$
v \leq c_{1}, \quad \text { for some } \quad c_{1} \geq 1,
$$

and satisfies the condition

$$
\langle F, \nu\rangle^{2} \leq C\left(1+|F|^{2}\right)^{1-\delta},
$$

for some $\delta>0$ and $C<\infty$, then the rescaled surface is self-similar as $t \rightarrow \infty$. They also show that this convergence is exponential in time.

For the rescaled surfaces denoted by $\tilde{M}_{s}=\tilde{F}(\cdot, s)(M)$, our main result states:

Main Theorem. Suppose $M_{0}$ satisfies the linear growth condition (1.5) and has bounded curvature. If in addition it satisfies

$$
\langle x, \nu\rangle^{2} \leq c \frac{1+|x|^{2}-u^{2}}{\log ^{\delta}\left(\mathrm{e}+|x|^{2}\right)},
$$

for some constant $c<\infty$ and some power $\delta>0$ then the solutions $\tilde{M}_{s}$ of the rescaled mean curvature flow (1.4) converge as $s \rightarrow \infty$ to a limiting surface $\tilde{M}_{\infty}$ which is self-similar, i.e., it satisfies

$$
F^{\perp}=\vec{H} .
$$




\section{Height estimate}

We define the height of $M_{t}$ with respect to the hyperplane $\mathbb{R}^{n} \times\{0\}$ by

$$
u(p, t)=\left\langle x(p, t), \mathrm{e}_{n+1}\right\rangle .
$$

In particular we have the following lemma, since $x(p, t)$ is the solution to mean curvature flow and so

$$
\left(\frac{d}{d t}-\Delta\right) u=0
$$

Lemma 2.1. The function $\eta(x, t)$ given by

$$
\eta(x, t)=\mathrm{e}+|x|^{2}-u^{2}+(2 n+m) t
$$

satisfies

$$
\left(\frac{d}{d t}-\Delta\right) \eta=2|\nabla u|^{2}+m
$$

for some constant $m$.

Now we would like to study $M_{t}$, a smooth solution to (1.1), which grows logarithmically. We would like to show that height $u(\cdot, t)$ satisfies the same logarithmic growth estimates as $u(\cdot, 0)$. Note in particular that the nonnegative function $|x|^{2}-u^{2}$ measures distance in the hyperplane orthogonal to $\mathrm{e}_{n+1}$. Our proposition then states:

Proposition 2.1. If for some negative constant $-\infty<c_{0} \leq 0$ and positive power $\delta \geq 1$, the inequality

$$
\frac{u^{2}}{\mathrm{e}+|x|^{2}-u^{2}}-1 \leq \frac{c_{0}}{\log ^{\delta}\left(\mathrm{e}+|x|^{2}-u^{2}\right)}
$$

is satisfied on $M_{0}$, then for all $t>0$,

$$
\frac{u^{2}}{\mathrm{e}+|x|^{2}-u^{2}+(2 n+m) t}-1 \leq \frac{c_{0}}{\log ^{\delta}\left(\mathrm{e}+|x|^{2}-u^{2}+(2 n+m) t\right)},
$$

and a positive constant $m \geq 4(\delta-1)$. 
Proof idea. The proof involves calculating the evolution of

$$
\left(\frac{u^{2}}{\mathrm{e}+|x|^{2}-u^{2}+(2 n+m) t}-1\right) \log ^{\delta}\left(\mathrm{e}+|x|^{2}-u^{2}+(2 n+m) t\right)
$$

and then using the weak maximum principle to obtain the result.

Thus when we have for our particular case

$$
f(\eta)=\log (\eta), \quad f^{\prime}(\eta)=\frac{1}{\eta}, \quad f^{\prime \prime}(\eta)=-\frac{1}{\eta^{2}}
$$

then the above lemma gives:

$$
\begin{aligned}
\left(\frac{d}{d t}-\Delta\right)\left(\frac{u^{2} f^{\delta}(\eta)}{\eta}-f^{\delta}(\eta)\right)= & \left(\frac{u^{2}}{\eta}-1\right) \delta f^{\delta-1}\left(\frac{2|\nabla u|^{2}+m}{\eta}+\frac{|\nabla \eta|^{2}}{\eta^{2}}\right) \\
& -\left(\frac{u^{2}}{\eta}-1\right) \delta(\delta-1) \frac{f^{\delta-2}}{\eta^{2}}|\nabla \eta|^{2} \\
& -2 \frac{f^{\delta}}{\eta}|\nabla u|^{2}-\frac{u^{2} f^{\delta}}{\eta^{2}}\left(2|\nabla u|^{2}+m\right) \\
& -2 \frac{u^{2} f^{\delta}}{\eta^{3}}|\nabla \eta|^{2}+2 \delta \frac{u^{2} f^{\delta-1}}{\eta^{3}}|\nabla \eta|^{2} \\
& -4 \delta \frac{u f^{\delta-1}}{\eta^{2}} \nabla u \cdot \nabla \eta+4 \frac{u f^{\delta}}{\eta^{2}} \nabla u \cdot \nabla \eta .
\end{aligned}
$$

Using Young's inequality we obtain:

$$
\left|4 \frac{u f^{\delta}}{\eta^{2}} \nabla u \cdot \nabla \eta\right| \leq 2 \frac{f^{\delta}}{\eta}|\nabla u|^{2}+2 \frac{u^{2} f^{\delta}}{\eta^{3}}|\nabla \eta|^{2} .
$$

Also note that in terms of a local orthonormal frame $\left\{e_{i}\right\}_{1 \leq i \leq n}$ on $M$ we have

$$
\nabla_{i} u=\nabla_{i}\left\langle x, \mathrm{e}_{n+1}\right\rangle=\left\langle\mathrm{e}_{i}, \mathrm{e}_{n+1}\right\rangle
$$

which implies that

$$
\nabla_{i} \eta=2|x| \frac{\left\langle x, \mathrm{e}_{i}\right\rangle}{|x|}-2\left\langle x, \mathrm{e}_{n+1}\right\rangle\left\langle\mathrm{e}_{i}, \mathrm{e}_{n+1}\right\rangle=2\left\langle\mathrm{e}_{i}, x-\left\langle x, \mathrm{e}_{n+1}\right\rangle \mathrm{e}_{n+1}\right\rangle
$$


so that:

$$
\begin{aligned}
|\nabla \eta|^{2} & =4 \sum_{i}\left(\left\langle x, \mathrm{e}_{i}\right\rangle-\left\langle x, \mathrm{e}_{n+1}\right\rangle\left\langle\mathrm{e}_{i}, \mathrm{e}_{n+1}\right\rangle\right)^{2} \\
& =4 \sum_{i}\left(\left\langle x, \mathrm{e}_{i}\right\rangle^{2}-2\left\langle x, \mathrm{e}_{n+1}\right\rangle\left\langle\mathrm{e}_{i}, \mathrm{e}_{n+1}\right\rangle\left\langle x, \mathrm{e}_{i}\right\rangle+\left\langle x, \mathrm{e}_{n+1}\right\rangle^{2}\left\langle\mathrm{e}_{i}, \mathrm{e}_{n+1}\right\rangle^{2}\right) \\
& =4\left(|x|^{2}-\langle\nu, x\rangle^{2}-2\left\langle x, \mathrm{e}_{n+1}\right\rangle^{2}+u^{2}\left(1-\left\langle\nu, \mathrm{e}_{n+1}\right\rangle^{2}\right)\right) \\
(2.2) \quad & \leq 4 \eta
\end{aligned}
$$

Also by the first derivative test for an extrema, we have at such a point $\nabla\left(u^{2} f^{\delta} / \eta-f^{\delta}\right)=0$. We expand this out to obtain:

$$
\begin{aligned}
0= & \nabla\left(\frac{u^{2} f^{\delta}(\eta)}{\eta}-f^{\delta}(\eta)\right)=2 \frac{u f^{\delta} \nabla u}{\eta}+\delta \frac{u^{2} f^{\delta-1} \nabla \eta}{\eta^{2}} \\
& -\frac{u^{2} f^{\delta} \nabla \eta}{\eta^{2}}-\delta \frac{f^{\delta-1} \nabla \eta}{\eta},
\end{aligned}
$$

which we rearrange to obtain:

$$
\begin{aligned}
2 \delta \frac{u^{2} f^{\delta-1}}{\eta^{3}}|\nabla \eta|^{2}-4 \delta \frac{u f^{\delta-1}}{\eta^{2}} \nabla u \cdot \nabla \eta & =-2 \delta^{2} \frac{f^{\delta-2}|\nabla \eta|^{2}}{\eta^{2}}+2 \delta^{2} \frac{u^{2} f^{\delta-2}|\nabla \eta|^{2}}{\eta^{3}} \\
& \leq-2 \delta^{2} \frac{f^{\delta-2}|\nabla \eta|^{2}}{\eta^{2}}+2 \delta^{2} \frac{f^{\delta-2}|\nabla \eta|^{2}}{\eta^{2}} \\
& =0 .
\end{aligned}
$$

The last inequality above follows if we assume that initially

$$
\frac{u^{2}}{\eta} \leq 1
$$

which is preserved during mean curvature flow as shown by the following adaption of Proposition 2.2 of Ecker and Huisken in [1]:

Proposition 2.2 (A priori height estimate). If for some $c_{0}<\infty$, the inequality

$$
\frac{u^{2}}{1+|x|^{2}-u^{2}} \leq C_{0}
$$

is satisfied on $M_{0}$, then for all $t>0$, and some constant $m \geq 0$,

$$
\frac{u^{2}}{1+|x|^{2}-u^{2}+(2 n+m) t} \leq C_{0} .
$$


Proof. We calculate the evolution equation

$$
\begin{aligned}
\left(\frac{d}{d t}-\Delta\right) \frac{u^{2}}{\eta}= & -2 \frac{|\nabla u|^{2}}{\eta}-\frac{u^{2}}{\eta^{2}}\left(2|\nabla u|^{2}+m\right)-2 u^{2} \frac{|\nabla \eta|^{2}}{\eta^{3}} \\
& +4 u \frac{\nabla \eta \cdot \nabla u}{\eta^{2}} .
\end{aligned}
$$

By Young's inequality we have:

$$
\left|4 u \frac{\nabla \eta \cdot \nabla u}{\eta^{2}}\right| \leq 2 \frac{|\nabla u|^{2}}{\eta}+2 u^{2} \frac{|\nabla \eta|^{2}}{\eta^{3}}
$$

which then implies that

$$
\left(\frac{d}{d t}-\Delta\right) \frac{u^{2}}{\eta} \leq-\frac{u^{2}}{\eta^{2}}\left(2|\nabla u|^{2}+m\right) \leq 0 .
$$

Therefore by the weak maximum principle [1] the result follows.

We are now ready to prove our proposition, namely if $M_{t}$ is a smooth solution of mean curvature flow and if initially $M_{0}$ converges to its tangent cone logarithmically, then such a rate is preserved during the evolution. In other words the solutions remain in the same growth class they started in.

Proof of Proposition 2.1. By using the inequalities (2.1) and (2.3) we obtain from the evolution equation for some positive power $\delta$, the following inequality at the maximum point:

$$
\begin{aligned}
\left(\frac{d}{d t}-\Delta\right)\left(\frac{u^{2}}{\eta}-1\right) f^{\delta} \leq & -\delta\left(1-\frac{u^{2}}{\eta}\right) f^{\delta-1}\left(\frac{2|\nabla u|^{2}+m}{\eta}+\frac{|\nabla \eta|^{2}}{\eta^{2}}\right) \\
& +\delta(\delta-1)\left(1-\frac{u^{2}}{\eta}\right) \frac{f^{\delta-2}}{\eta^{2}}|\nabla \eta|^{2} \\
& -\frac{u^{2} f^{\delta}}{\eta^{2}}\left(2|\nabla u|^{2}+m\right) .
\end{aligned}
$$

Note that since $u^{2} / \eta-1$ by our initial assumption is negative, the only positive term we have in the above inequality is

$$
\delta(\delta-1)\left(1-\frac{u^{2}}{\eta}\right) \frac{f^{\delta-2}}{\eta^{2}}|\nabla \eta|^{2} \leq 4 \delta(\delta-1)\left(1-\frac{u^{2}}{\eta}\right) \frac{f^{\delta-2}}{\eta},
$$


since by $(2.2)|\nabla \eta|^{2} \leq 4 \eta$. Now we will choose a positive constant $m$ so that we can control this term. In particular we see that $m$ must be chosen so that

$$
\delta\left(1-\frac{u^{2}}{\eta}\right) \frac{f^{\delta-1}}{\eta}\left(4 \frac{\delta-1}{f}-m\right) \leq 0 .
$$

Thus since $f(\eta) \geq \log e=1$, if we choose $m \geq 4(\delta-1)$, we can drop this remaining negative term to obtain

$$
\left(\frac{d}{d t}-\Delta\right)\left(\frac{u^{2}}{\eta}-1\right) f^{\delta} \leq 0 .
$$

Therefore once again by the weak maximum principle [1], the result we need is obtained.

\section{Curvature estimates}

In [1] Ecker and Huisken obtaining time and polynomial spatial decay estimates for the curvature, and in this section we show that initial logarithmic spatial decay behaviour is also preserved during the course of the evolution. If we consider the scaled solutions $\left(M_{s}^{\rho}\right)_{s \in(0,1)}$, where

$$
M_{s}^{\rho}=\frac{1}{\rho} M_{\rho^{2} s},
$$

then the second fundamental form of $\left(M_{s}^{\rho}\right)$ given by $A_{\rho}$ satisfies a scaling property which we obtain by setting $x=\rho y$ and $t=\rho^{2} s$. We then have for $x \in M_{t}$ and $y \in M_{s}^{\rho}$ that

$$
\left|\nabla^{m} A_{\rho}(y)\right|^{2}=\rho^{2(m+1)}\left|\nabla^{m} A(x)\right|^{2},
$$

so that the statements

$$
\left|\nabla^{m} A_{\rho}(y)\right|^{2} \leq c_{2}(m)
$$

for $y \in M_{s}^{\rho} \cap B_{1 / 2}, s \in(3 / 4,1)$ and

$$
\left|\nabla^{m} A(x)\right|^{2} \leq \frac{c_{2}(m)}{\rho^{2(m+1)}},
$$

for $x \in M_{t} \cap B_{\rho / 2}, t \in\left(3 / 4 \rho^{2}, \rho^{2}\right)$ are equivalent. In view of this we propose the following proposition which satisfies the correct scaling of the second fundamental form: 
Proposition 3.1. Let $M_{t}$ be a smooth solution of (1.1), satisfying $v \leq c_{1}$, and the additional assumption

$$
\left|\nabla^{m} A\right|^{2} \leq c_{2}(m) \frac{\log ^{\delta(m+1)}\left(\mathrm{e}+|x|^{2}\right)}{\left(\mathrm{e}+|x|^{2}\right)^{m+1}}
$$

at time $t=0, m \geq 0$ and $\delta \geq 0$. Then for all $t>0$

$$
\left|\nabla^{m} A\right|^{2} \leq C_{m} \frac{\log ^{\delta(m+1)}\left(\mathrm{e}+\left(\sqrt{|x|^{2}+2 n t}-\sqrt{\beta t}\right)^{2}\right)}{\left(\mathrm{e}+\left(\sqrt{|x|^{2}+2 n t}-\sqrt{\beta t}\right)^{2}\right)^{m+1}},
$$

where $\beta=\beta\left(c_{1}\right)>0$ and $C_{m}=C_{m}\left(n, m, c_{1}, c_{2}(0), \ldots, c_{2}(m)\right)$.

Proof idea. Let $g=|A|^{2} v^{2} f(\eta)+L v^{2}$ where $f(\eta)$ is an arbitrary non-negative function and $L>0$ to be determined later. For the case $m=0$ we thus have:

$$
\begin{aligned}
\left(\frac{d}{d t}-\Delta\right) g= & f(\eta)\left(\frac{d}{d t}-\Delta\right)|A|^{2} v^{2}+|A|^{2} v^{2}\left(\frac{d}{d t}-\Delta\right) f(\eta) \\
& -2 \nabla f(\eta) \cdot \nabla\left(|A|^{2} v^{2}\right)+L\left(\frac{d}{d t}-\Delta\right) v^{2} \\
\leq & -2 \frac{f(\eta)}{v} \nabla v \cdot \nabla\left(|A|^{2} v^{2}\right)+|A|^{2} v^{2}\left(\frac{d}{d t}-\Delta\right) f(\eta) \\
& -2 \nabla f(\eta) \cdot \nabla\left(|A|^{2} v^{2}\right)-2 L\left(|A|^{2} v^{2}-3|\nabla v|^{2}\right) .
\end{aligned}
$$

Note that we have

$$
\nabla g=f(\eta) \nabla\left(|A|^{2} v^{2}\right)+|A|^{2} v^{2} \nabla f(\eta)+2 v L \nabla v,
$$

so that we if we multiply both sides by $-2(\nabla v) / v$ we end up with:

$$
-\frac{2}{v} \nabla v \cdot \nabla g=-2 \frac{f}{v} \nabla v \cdot \nabla\left(|A|^{2} v^{2}\right)-2|A|^{2} v \nabla v \cdot \nabla f-4 L|\nabla v|^{2} .
$$

Similarly multiplying both sides by $-2(\nabla f) / f$ gives:

$$
-\frac{2}{f} \nabla f \cdot \nabla g=-2 \nabla f \cdot \nabla\left(|A|^{2} v^{2}\right)-2 \frac{|\nabla f|^{2}}{f}|A|^{2} v^{2}-4 L \frac{v}{f} \nabla v \cdot \nabla f .
$$


Substituting the above equations into the estimate thus gives

$$
\begin{aligned}
\left(\frac{d}{d t}-\Delta\right) g \leq & -2\left(\frac{\nabla v}{v}+\frac{\nabla f}{f}\right) \cdot \nabla g+|A|^{2} v^{2}\left(\frac{2}{v} \nabla f \cdot \nabla v+\frac{2}{f}|\nabla f|^{2}\right. \\
& \left.+\left(\frac{d}{d t}-\Delta\right) f-2 L\right)+4 L \frac{v}{f} \nabla v \cdot \nabla f-2 L|\nabla v|^{2}
\end{aligned}
$$

By Young's inequality we have that

$$
\left|4 L \frac{v}{f} \nabla v \cdot \nabla f\right| \leq 2 L \frac{v^{2}}{f^{2}}|\nabla f|^{2}+2 L|\nabla v|^{2},
$$

and we estimate the vector $2 v^{-1} \nabla v$ using the inequality $v^{-1}|\nabla v| \leq|A| v$, and from the Proposition for the long-time existence for the case $m=0$ and the fact $C(0)=c_{1}^{2} / 2$. This together then implies that

$$
\frac{2}{v} \nabla v \cdot \nabla f \leq c_{1} \sqrt{\frac{2}{t}}|\nabla f| .
$$

Thus we finally have the estimate

$$
\begin{aligned}
\left(\frac{d}{d t}-\Delta\right) g \leq & -2\left(\frac{\nabla v}{v}+\frac{\nabla f}{f}\right) \cdot \nabla g+|A|^{2} v^{2}\left(c_{1} \sqrt{\frac{2}{t}}|\nabla f|+\frac{2}{f}|\nabla f|^{2}\right. \\
& \left.+\left(\frac{d}{d t}-\Delta\right) f-2 L\right)+2 L \frac{v^{2}}{f^{2}}|\nabla f|^{2}
\end{aligned}
$$

Now we define

$$
\eta(x, t)=\mathrm{e}+\left(\sqrt{|x|^{2}+2 n t}-\sqrt{\beta t}\right)^{2}
$$

where $\beta>0$ will be chosen later. Recall that we have the inequality $(2.2)$

$$
|\nabla \eta|^{2} \leq 4 \eta
$$

and also in view of the fact that $(d / d t-\Delta)\left(|x|^{2}+2 n t\right)=0$ we have

$$
\begin{aligned}
\left(\frac{d}{d t}-\Delta\right) \eta= & 2\left(\sqrt{|x|^{2}+2 n t}-\sqrt{\beta t}\right)\left(\frac{d}{d t}-\Delta\right) \sqrt{|x|^{2}+2 n t} \\
& -2\left|\nabla \sqrt{|x|^{2}+2 n t}\right|^{2}+\beta-\sqrt{\frac{\beta}{t}\left(|x|^{2}+2 n t\right)} \\
= & \left(\sqrt{|x|^{2}+2 n t}-\sqrt{\beta t}\right) \frac{\left.\left.|\nabla| x\right|^{2}\right|^{2}}{2\left(|x|^{2}+2 n t\right)^{3 / 2}}
\end{aligned}
$$




$$
\begin{aligned}
& -\frac{\left.\left.|\nabla| x\right|^{2}\right|^{2}}{2\left(|x|^{2}+2 n t\right)}+\beta-\sqrt{\frac{\beta}{t}\left(|x|^{2}+2 n t\right)} \\
= & \frac{\left.\left.|\nabla| x\right|^{2}\right|^{2}}{2\left(|x|^{2}+2 n t\right)}-\sqrt{\beta t} \frac{\left.\left.|\nabla| x\right|^{2}\right|^{2}}{2\left(|x|^{2}+2 n t\right)^{3 / 2}} \\
& -\frac{\left.\left.|\nabla| x\right|^{2}\right|^{2}}{2\left(|x|^{2}+2 n t\right)}+\beta-\sqrt{\frac{\beta}{t}\left(|x|^{2}+2 n t\right)} \\
\leq & \beta-\sqrt{\frac{\beta}{t}\left(|x|^{2}+2 n t\right)} .
\end{aligned}
$$

Proof of Proposition 3.1. As stated above, for the case $m=0$ if $g=$ $|A|^{2} v^{2} f(\eta)+L v^{2}$, where $f(\eta)$ is an arbitrary non-negative function and $L>0$ to be determined later, we have the evolution equation of $g$ given by (3.1). Now we define

$$
f(\eta(x, t))=\frac{\eta(x, t)}{\log ^{\delta} \eta(x, t)}, \quad f^{\prime}(\eta)=\frac{\log \eta-\delta}{\log ^{\delta+1} \eta}, \quad f^{\prime \prime}(\eta)=\frac{\delta(1+\delta-\log \eta)}{\eta \log ^{\delta+2} \eta}
$$

and for some $\beta>0$ to be chosen later and $\eta(x, t)$ whose evolution equation is given by $(3.2)$.

Thus we begin by estimating the terms in (3.1) by first calculating the evolution of $f(\eta)$ :

$$
\begin{aligned}
\left(\frac{d}{d t}-\Delta\right) f(\eta)= & \eta\left(\frac{d}{d t}-\Delta\right) \log ^{-\delta} \eta+\log ^{-\delta} \eta\left(\frac{d}{d t}-\Delta\right) \eta \\
& -2 \nabla \eta \cdot \nabla \log ^{-\delta} \eta \\
= & -\delta \log ^{-\delta-1} \eta\left(\frac{d}{d t}-\Delta\right) \eta-\frac{\delta}{\eta}|\nabla \eta|^{2} \log ^{-\delta-1} \eta \\
& -\frac{\delta(\delta+1)}{\eta}|\nabla \eta|^{2} \log ^{-\delta-2} \eta+\log ^{-\delta} \eta\left(\frac{d}{d t}-\Delta\right) \eta \\
& +2 \frac{\delta}{\eta}|\nabla \eta|^{2} \log ^{-\delta-1} \eta \\
= & \left(1-\frac{\delta}{\log \eta}\right) \log ^{-\delta} \eta\left(\frac{d}{d t}-\Delta\right) \eta \\
& +\delta\left(1-\frac{\delta+1}{\log \eta}\right) \frac{|\nabla \eta|^{2}}{\eta} \log ^{-\delta-1} \eta \\
\leq & \left(\frac{d}{d t}-\Delta\right) \eta+4 \delta \leq 4 \delta+\beta-\sqrt{\frac{\beta}{t}\left(|x|^{2}+2 n t\right)}
\end{aligned}
$$


where we use the inequality $(2.2)|\nabla \eta|^{2} \leq 4 \eta$ and the fact that $\log \eta \geq 1$. The next term we estimate is:

$$
\begin{aligned}
\frac{2}{f}|\nabla f|^{2}= & 2 \frac{f}{\eta^{2}}|\nabla \eta|^{2}+2 \delta^{2} \frac{f}{\eta^{4} \log ^{2} \eta}|\nabla \eta|^{2} \\
& -4 \delta \frac{f}{\eta^{3} \log \eta}|\nabla \eta|^{2} \\
\leq & 8 \frac{f}{\eta}+8 \delta^{2} \frac{f}{\eta^{3} \log ^{2} \eta} \leq 8\left(1+\delta^{2} / \mathrm{e}^{2}\right),
\end{aligned}
$$

which also gives us that

$$
2 L \frac{v^{2}}{f^{2}}|\nabla f|^{2} \leq 8\left(1+\delta^{2} / \mathrm{e}^{2}\right) L \frac{v^{2}}{f} .
$$

Finally we estimate

$$
|\nabla f| \leq|\nabla \eta| \log ^{-\delta} \eta-\frac{\delta}{\eta}|\nabla \eta| \log ^{-\delta-1} \eta \leq|\nabla \eta| \leq 2 \sqrt{|x|^{2}+2 n t}+2 \sqrt{\beta t},
$$

which we use to obtain

$$
c_{1} \sqrt{\frac{2}{t}}|\nabla f| \leq c_{1} \sqrt{\frac{2}{t}}|\nabla \eta| \leq 2 c_{1} \sqrt{\frac{2}{t}\left(|x|^{2}+2 n t\right)}+2 c_{1} \sqrt{2 \beta} .
$$

We therefore have the final estimate for $g$ by substituting the above estimates into (3.1):

$$
\begin{aligned}
\left(\frac{d}{d t}-\Delta\right) g \leq & -2\left(\frac{\nabla v}{v}+\frac{\nabla f}{f}\right) \cdot \nabla g+|A|^{2} v^{2}\left(2 c_{1} \sqrt{2 \beta}+\beta+4 \delta\right. \\
& \left.+8\left(1+\delta^{2} / \mathrm{e}^{2}\right)-2 L\right)-\sqrt{\frac{|x|^{2}+2 n t}{t}}\left(\sqrt{\beta}-2 \sqrt{2} c_{1}\right)|A|^{2} v^{2} \\
& +8\left(1+\delta^{2} / \mathrm{e}^{2}\right) L \frac{v^{2}}{f(\eta)} \\
\leq & \vec{b} \cdot \nabla g+|A|^{2} v^{2}\left(2 c_{1} \sqrt{2 \beta}+\beta+4 \delta+8\left(1+\delta^{2} / \mathrm{e}^{2}\right)-2 L\right) \\
& +8\left(1+\delta^{2} / \mathrm{e}^{2}\right) L \frac{v^{2}}{f(\eta)}
\end{aligned}
$$

for some large enough $\beta=\beta\left(c_{1}\right)$, where we define

$$
\vec{b}=-2\left(\frac{\nabla v}{v}+\frac{\nabla f}{f}\right) .
$$


If we now choose $L$ large depending on $\beta, c_{1}$ and $\delta$, and define $k=\sup _{M_{0}} g+$ $9\left(1+\delta^{2} / \mathrm{e}^{2}\right) L c_{1}^{2}$, we obtain

$$
\left(\frac{d}{d t}-\Delta\right) g \leq \vec{b} \cdot \nabla g-\frac{g-k}{f(\eta)},
$$

where we have used the estimate $v(x, t) \leq c_{1}$ once again. Now let $g_{k}=$ $\max (g-k, 0)$, and since $g_{k} \cdot(g-k)=g_{k}^{2}$, we obtain the result using the maximum principle with $g_{k}^{2}$.

For the case $m=1$, we compute as in the previous proposition the evolution

$$
\begin{aligned}
\left(\frac{d}{d t}-\Delta\right)|\nabla A|^{2} f^{2}(\eta)= & f^{2}\left(\frac{d}{d t}-\Delta\right)|\nabla A|^{2}+|\nabla A|^{2}\left(\frac{d}{d t}-\Delta\right) f^{2}(\eta) \\
& -2 \nabla f^{2} \cdot \nabla|\nabla A|^{2} \\
\leq & c(n)|A|^{2}|\nabla A|^{2} f^{2}(\eta)-2\left|\nabla^{2} A\right|^{2} f^{2}(\eta) \\
& +8\left(\frac{\log \eta-\delta}{\log ^{\delta+1} \eta}\right)^{2}|\nabla \eta|^{2}|\nabla A|^{2}+2\left|\nabla^{2} A\right|^{2} f^{2}
\end{aligned}
$$

Since by $(2.2)|\nabla \eta|^{2} \leq 4 \eta$ and $|A|^{2} f(\eta) \leq C_{0}$ (recall that $v \leq 1$ ) we estimate

$$
\left(\frac{d}{d t}-\Delta\right)|\nabla A|^{2} f^{2}(\eta) \leq c\left(n, \delta, C_{0}\right)|\nabla A|^{2} f(\eta) .
$$

Similarly we derive

$$
\left(\frac{d}{d t}-\Delta\right)|A|^{2} f(\eta) \leq-|\nabla A|^{2} f(\eta)+c\left(n, \delta, C_{0}\right)|A|^{2} .
$$

Also recall that for $v \geq 1$ one has

$$
\left(\frac{d}{d t}-\Delta\right) v^{2} \leq-2|A|^{2}
$$

so that if we choose large enough positive constants $K$ and $L$ depending on $n, \delta$ and $C_{0}$ we have that

$$
\left(\frac{d}{d t}-\Delta\right)\left(|\nabla A|^{2} f^{2}(\eta)+K|A|^{2} f(\eta)+L v^{2}\right) \leq 0 .
$$

The proposition for $m=1$ then follows from the maximum principle. We iterate over $m$ similarly to prove the general statement. 


\section{Asymptotic behaviour}

As our initial graph evolves under mean curvature flow, it will move off to infinity with speed proportional to $1 / \sqrt{t}$ and height proportional to $\sqrt{t}$, so studying its global shape as time goes to infinity will give us no insight, unless we rescale the surfaces back and prevent it from diverging to infinity.

We therefore define the following rescaling:

$$
\tilde{F}(s)=\frac{F(t)}{\sqrt{2 t+1}},
$$

where the new time variable is given by

$$
s=\frac{1}{2} \log (2 t+1)
$$

for $0 \leq s<\infty$. The rescaled mean curvature flow then becomes (1.4) with the same initial condition

$$
\tilde{F}(\cdot, 0)=F(\cdot, 0)
$$

Now for the rescaled surfaces denoted by $\tilde{M}_{s}=\tilde{F}(\cdot, s)(M)$ we have the following result:

Main Theorem. Suppose $M_{0}$ satisfies the linear growth condition (1.5) and has bounded curvature. If in addition it satisfies

$$
\langle x, \nu\rangle^{2} \leq c \frac{1+|x|^{2}-u^{2}}{\log ^{\delta}\left(\mathrm{e}+|x|^{2}\right)}
$$

for some constant $c<\infty$ and some power $\delta>0$ then the solutions $\tilde{M}_{s}$ of the rescaled mean curvature flow (1.4) converge as $s \rightarrow \infty$ to a limiting surface $\tilde{M}_{\infty}$ which is self-similar, i.e., it satisfies

$$
F^{\perp}=\vec{H}
$$

Before we prove this we show that the up to a time dependent factor, the condition (4.1) is preserved for all time. 
Lemma 4.1. Suppose our initial graph $M_{0}$ has bounded gradient and curvature and we have

$$
\langle x, \nu\rangle^{2} \leq c \frac{1+|x|^{2}-u^{2}}{\log ^{\delta}\left(\mathrm{e}+|x|^{2}\right)},
$$

for some constant $c<\infty$ and positive $\delta \geq 0$, then for all $t>0, M_{t}$ also satisfies

$$
\langle x, \nu\rangle^{2} \leq c(t) \frac{1+|x|^{2}-u^{2}+2 n t}{\log ^{\delta}\left(\mathrm{e}+|x|^{2}+2 n t\right)} .
$$

Proof. Let $f=\langle x, \nu\rangle$, then we have

$$
\begin{aligned}
\left(\frac{d}{d t}-\Delta\right) f^{2} & =2 f^{2}|A|^{2}-4 H f-2|\nabla f|^{2} \\
& \leq C\left(f^{2}+1\right)-2|\nabla f|^{2}
\end{aligned}
$$

Also if we define

$$
\eta_{1}=\mathrm{e}+|x|^{2}+2 n t \quad \text { and } \quad \eta_{2}=1+|x|^{2}-u^{2}+2 n t
$$

then by the product-rule for the heat operator,

$$
\begin{aligned}
\left(\frac{d}{d t}-\Delta\right) \log ^{\delta} \eta_{1} & =\delta \frac{\left|\nabla \eta_{1}\right|^{2}}{\eta_{1}^{2}} \log ^{\delta-1} \eta_{1}-\delta(\delta-1) \frac{\left|\nabla \eta_{1}\right|^{2}}{\eta_{1}^{2}} \log ^{\delta-2} \eta_{1} \\
& =\delta\left(\frac{1}{\log \eta_{1}}-\frac{\delta-1}{\log ^{2} \eta_{1}}\right) \frac{\left|\nabla \eta_{1}\right|^{2}}{\eta_{1}^{2}} \log ^{\delta} \eta_{1} \\
& \leq 4 \delta\left(\frac{1}{\log \eta_{1}}-\frac{\delta-1}{\log ^{2} \eta_{1}}\right) \frac{1}{\eta_{1}} \log ^{\delta} \eta_{1} \leq C \log ^{\delta} \eta_{1}
\end{aligned}
$$

where we have used the inequality $\left|\nabla \eta_{1}\right|^{2} \leq 4 \eta_{1}$ and denoted all constants which depend on the curvature bound and $t$ by $C$. Similarly we have

$$
\left(\frac{d}{d t}-\Delta\right) \eta_{2}^{-1}=-2 \frac{\left|\nabla \eta_{2}\right|^{2}}{\eta_{2}^{3}}-2 \frac{|\nabla u|^{2}}{\eta_{2}^{2}}
$$


so that

$$
\begin{aligned}
\left(\frac{d}{d t}-\Delta\right) \frac{f^{2}}{\eta_{2}}= & \frac{1}{\eta_{2}}\left(\frac{d}{d t}-\Delta\right) f^{2}+f^{2}\left(\frac{d}{d t}-\Delta\right) \frac{1}{\eta_{2}}-2 \nabla f^{2} \cdot \nabla \frac{1}{\eta_{2}} \\
\leq & \frac{C}{\eta_{2}}\left(f^{2}+1\right)-2 \frac{|\nabla f|^{2}}{\eta_{2}}-2 f^{2} \frac{\left|\nabla \eta_{2}\right|^{2}}{\eta_{2}^{3}}-2 f^{2} \frac{|\nabla u|^{2}}{\eta_{2}^{2}} \\
& +4 \frac{f}{\eta_{2}^{2}} \nabla f \cdot \nabla \eta_{2} \\
\leq & \frac{C}{\eta_{2}}\left(f^{2}+1\right)-2 f^{2} \frac{|\nabla u|^{2}}{\eta_{2}^{2}} \leq \frac{C}{\eta_{2}}\left(f^{2}+1\right),
\end{aligned}
$$

where we have used Young's inequality

$$
\left|4 \frac{f}{\eta_{2}^{2}} \nabla f \cdot \nabla \eta_{2}\right| \leq 2 \frac{|\nabla f|^{2}}{\eta_{2}}+2 f^{2} \frac{\left|\nabla \eta_{2}\right|^{2}}{\eta_{2}^{3}}
$$

Therefore since $\eta_{2}^{-1} \log ^{\delta} \eta_{1} \leq c$, some constant $c>0$ we have:

$$
\begin{aligned}
\left(\frac{d}{d t}-\Delta\right) \frac{f^{2}}{\eta_{2}} \log ^{\delta} \eta_{1}= & \frac{f^{2}}{\eta_{2}}\left(\frac{d}{d t}-\Delta\right) \log ^{\delta} \eta_{1}+\log ^{\delta} \eta_{1}\left(\frac{d}{d t}-\Delta\right) \frac{f^{2}}{\eta_{2}} \\
& -2 \nabla \frac{f^{2}}{\eta_{2}} \cdot \nabla \log ^{\delta} \eta_{1} \\
\leq & C\left(\frac{f^{2}}{\eta_{2}} \log ^{\delta} \eta_{1}+1\right)-2 \nabla \frac{f^{2}}{\eta_{2}} \cdot \nabla \log ^{\delta} \eta_{1}
\end{aligned}
$$

Now we calculate

$$
-2 \nabla \frac{f^{2}}{\eta_{2}} \cdot \nabla \log ^{\delta} \eta_{1}=2 \delta \frac{f^{2} \log ^{\delta-1} \eta_{1}}{\eta_{1} \eta_{2}^{2}} \nabla \eta_{1} \cdot \nabla \eta_{2}-4 \delta \frac{f \log ^{\delta-1} \eta_{1}}{\eta_{1} \eta_{2}} \nabla f \cdot \nabla \eta_{1},
$$

and once again by Young's inequality we can estimate the first term by

$$
\begin{aligned}
\left|2 \delta \frac{f^{2} \log ^{\delta-1} \eta_{1}}{\eta_{1} \eta_{2}^{2}} \nabla \eta_{1} \cdot \nabla \eta_{2}\right| & \leq \frac{f^{2}}{\eta_{2}} \log ^{\delta} \eta_{1}\left(4 \delta^{2} \frac{\left|\nabla \eta_{1}\right|^{2}}{\eta_{1}^{2} \log ^{2} \eta_{1}}+\frac{\left|\nabla \eta_{2}\right|^{2}}{\eta_{2}^{2}}\right) \\
& \leq C \frac{f^{2}}{\eta_{2}} \log ^{\delta} \eta_{1}
\end{aligned}
$$


since $\left|\nabla \eta_{1}\right|^{2} \leq 4 \eta_{1}$ and similarly $\left|\nabla \eta_{2}\right|^{2} \leq 4 \eta_{2}$. Thus dropping the negative term gives us the following estimate:

$$
\left(\frac{d}{d t}-\Delta\right) \frac{f^{2}}{\eta_{2}} \log ^{\delta} \eta_{1} \leq C\left(\frac{f^{2}}{\eta_{2}} \log ^{\delta} \eta_{1}+1\right),
$$

which by the maximum principle implies the result that we require.

In order to prove the Main Theorem we will need to know the evolution equation of a test-function in the rescaled case. The test function we use will have the following general form

$$
\tilde{\rho}(\tilde{x}, s)=g(\tilde{x}, s) h(s)
$$

where

$$
g(\tilde{x}, s)=\frac{\log ^{\delta}\left(\tilde{\eta}_{1}\right)}{\tilde{\eta}_{2}^{p}}
$$

for some positive powers $\delta>0$ and $p>0$ to be specified later.

The heat operator of $\tilde{\rho}$ is

$$
\left(\frac{d}{d s}-\tilde{\Delta}\right) \tilde{\rho}(\tilde{x}, s)=h(s)\left(\frac{d}{d s}-\tilde{\Delta}\right) g(\tilde{x}, s)+h^{\prime}(s) g(\tilde{x}, s)
$$

and since

$$
\begin{aligned}
\left(\frac{d}{d s}-\tilde{\Delta}\right) g(\tilde{x}, s)= & \frac{1}{\tilde{\eta}_{2}^{p}}\left(\frac{d}{d s}-\tilde{\Delta}\right) \log ^{\delta} \tilde{\eta}_{1}+\log ^{\delta} \tilde{\eta}_{1}\left(\frac{d}{d s}-\tilde{\Delta}\right) \frac{1}{\tilde{\eta}_{2}^{p}} \\
& -2 \nabla \log ^{\delta} \tilde{\eta}_{1}\left(\tilde{\eta}_{1}\right) \cdot \nabla \frac{1}{\tilde{\eta}_{2}^{p}} \\
= & \delta \frac{\log ^{\delta-1} \tilde{\eta}_{1}}{\tilde{\eta}_{2}^{p}}\left(\frac{d}{d s}-\tilde{\Delta}\right) \tilde{\eta}_{1}-\delta(\delta-1) \frac{\left|\nabla \tilde{\eta}_{1}\right|^{2}}{\tilde{\eta}_{1}^{2} \tilde{\eta}_{2}^{p}} \log ^{\delta-2} \tilde{\eta}_{1} \\
& -p \frac{\log ^{\delta} \tilde{\eta}_{1}}{\tilde{\eta}_{2}^{p+1}}\left(\frac{d}{d s}-\tilde{\Delta}\right) \tilde{\eta}_{2}-p(p+1) \frac{\left|\nabla \tilde{\eta}_{2}\right|^{2}}{\tilde{\eta}_{2}^{p+2}} \log ^{\delta} \tilde{\eta}_{1} \\
& -2 \nabla \log ^{\delta} \tilde{\eta}_{1} \cdot \nabla \tilde{\eta}_{2}^{-p},
\end{aligned}
$$

we obtain the following: 
Proposition 4.1. For twice differentiable functions $\tilde{\eta}_{1}(\tilde{x}, s)$ and $\tilde{\eta}_{2}(\tilde{x}, s)$ and continuous $h(s)$, such that

$$
\tilde{\rho}(\tilde{x}, s)=g(\tilde{x}, s) h(s)=\frac{\log ^{\delta} \tilde{\eta}_{1}}{\tilde{\eta}_{2}^{p}} h(s),
$$

for some positive powers $\delta>0$ and $p>0$, we have

$$
\begin{aligned}
\left(\frac{d}{d s}-\tilde{\Delta}\right) \tilde{\rho}(\tilde{x}, s) \leq & \delta \frac{\tilde{\rho}}{\log \tilde{\eta}_{1}}\left(\frac{d}{d s}-\tilde{\Delta}\right) \tilde{\eta}_{1}-\delta\left(\frac{\delta}{2}-1\right) \frac{\left|\nabla \tilde{\eta}_{1}\right|^{2}}{\tilde{\eta}_{1}^{2} \log ^{2} \tilde{\eta}_{1}} \tilde{\rho} \\
& -p \frac{\tilde{\rho}}{\tilde{\eta}_{2}}\left(\frac{d}{d s}-\tilde{\Delta}\right) \tilde{\eta}_{2}+p(p-1) \frac{\left|\nabla \tilde{\eta}_{2}\right|^{2}}{\tilde{\eta}_{2}^{2}} \tilde{\rho} \\
& +h^{\prime}(s) g(\tilde{x}, s) .
\end{aligned}
$$

Proof. The crucial ingredient of the proof comes from expanding the term

$$
-2 h(s) \nabla \log ^{\delta} \tilde{\eta}_{1} \cdot \nabla \tilde{\eta}_{2}^{-p}=2 \delta p \frac{\tilde{\rho}}{\tilde{\eta}_{1} \tilde{\eta}_{2} \log \tilde{\eta}_{1}} \nabla \tilde{\eta}_{1} \cdot \nabla \tilde{\eta}_{2},
$$

which by Peter-Paul's inequality gives:

$$
\left|2 \delta p \frac{\tilde{\rho}}{\tilde{\eta}_{1} \tilde{\eta}_{2} \log \tilde{\eta}_{1}} \nabla \tilde{\eta}_{1} \cdot \nabla \tilde{\eta}_{2}\right| \leq 2 p^{2} \frac{\left|\nabla \tilde{\eta}_{2}\right|^{2}}{\tilde{\eta}_{2}^{2}} \tilde{\rho}+\frac{1}{2} \delta^{2} \frac{\left|\nabla \tilde{\eta}_{1}\right|^{2}}{\tilde{\eta}_{1}^{2} \log ^{2} \tilde{\eta}_{1}} \tilde{\rho}
$$

Therefore since

$$
\begin{aligned}
\left(\frac{d}{d s}-\tilde{\Delta}\right) \tilde{\rho}(\tilde{x}, s)= & \delta \frac{\tilde{\rho}}{\log \tilde{\eta}_{1}}\left(\frac{d}{d s}-\tilde{\Delta}\right) \tilde{\eta}_{1}-\delta(\delta-1) \frac{\left|\nabla \tilde{\eta}_{1}\right|^{2}}{\tilde{\eta}_{1}^{2} \log ^{2} \tilde{\eta}_{1}} \tilde{\rho} \\
& -p \frac{\tilde{\rho}}{\tilde{\eta}_{2}}\left(\frac{d}{d s}-\tilde{\Delta}\right) \tilde{\eta}_{2}-p(p+1) \frac{\left|\nabla \tilde{\eta}_{2}\right|^{2}}{\tilde{\eta}_{2}^{2}} \tilde{\rho}+h^{\prime}(s) g(\tilde{x}, s) \\
& -2 \nabla \log ^{\delta} \tilde{\eta}_{1} \cdot \nabla \frac{h(s)}{\tilde{\eta}_{2}^{p}}
\end{aligned}
$$

we obtain our result by using the above estimate.

\subsection{Proof of the Main Theorem}

The result of the Main Theorem will follow from the following estimate for some $0<\gamma<2$ :

$$
\sup _{\tilde{M}_{s}} \frac{(\tilde{H}+\langle\tilde{x}, \tilde{\nu}\rangle)^{2} \tilde{v}^{2}}{\tilde{\eta}_{2}^{p} \log ^{-\epsilon} \tilde{\eta}_{1}} \leq(1+s)^{-\gamma} \sup _{M_{0}} \frac{(H+\langle x, \nu\rangle)^{2} v^{2}}{\eta_{2}^{p} \log ^{-\epsilon} \eta_{1}},
$$


where $0<\epsilon<\delta, 0<p<1$, and for some choice of test functions $\eta_{1}$ and $\eta_{2}$. Note that this implies polynomial convergence on compact subsets, instead of exponentially fast convergence, obtained in regard to the corresponding estimate of Ecker and Huisken.

We will make use of the following lemma from Ecker and Huisken [1]:

Lemma 4.2. The normalized quantity $\tilde{H}+\langle\tilde{x}, \tilde{\nu}\rangle$ satisfies the evolution equation

$$
\left(\frac{d}{d s}-\tilde{\Delta}\right)(\tilde{H}+\langle\tilde{x}, \tilde{\nu}\rangle)=\left(|\tilde{A}|^{2}-1\right)(\tilde{H}+\langle\tilde{x}, \tilde{\nu}\rangle) .
$$

Proof. To begin with note that $\tilde{H}=\psi^{-1}(t) H$ and $\langle\tilde{x}, \tilde{\nu}\rangle=\psi(t)\langle x, \nu\rangle$ where $\psi(t)=1 / \sqrt{2 t+1}$ is the rescaling factor. As in [2], we say that $H$ is of "degree" -1 and $\langle x, \nu\rangle$ is of "degree" 1 . This together with the evolution equations

$$
\left(\frac{d}{d t}-\Delta\right) H=|A|^{2} H \quad \text { and } \quad\left(\frac{d}{d t}-\Delta\right)\langle x, \nu\rangle=|A|^{2}\langle x, \nu\rangle-2 H,
$$

gives us

$$
\begin{aligned}
\left(\frac{d}{d s}-\tilde{\Delta}\right)(\tilde{H}+\langle\tilde{x}, \tilde{\nu}\rangle) & =|\tilde{A}|^{2}(\tilde{H}+\langle\tilde{x}, \tilde{\nu}\rangle)-2 \tilde{H}+\tilde{H}-\langle\tilde{x}, \tilde{\nu}\rangle \\
& =\left(|\tilde{A}|^{2}-1\right)(\tilde{H}+\langle\tilde{x}, \tilde{\nu}\rangle)
\end{aligned}
$$

which is the result we want.

Similarly we have

$$
\left(\frac{d}{d s}-\tilde{\Delta}\right) \tilde{v}^{2}=-2|\tilde{A}|^{2} \tilde{v}^{2}-6|\nabla \tilde{v}|^{2},
$$

which gives us the following inequality for $f^{2}=(\tilde{H}+\langle\tilde{x}, \tilde{\nu}\rangle)^{2} \tilde{v}^{2}$

$$
\left(\frac{d}{d s}-\tilde{\Delta}\right) f^{2} \leq-2 f^{2}-2 \frac{1}{\tilde{v}} \nabla \tilde{v} \cdot \nabla f^{2} .
$$

Multiplying this with a test function $\tilde{\rho}(\tilde{x}, s)$ we derive

$$
\begin{aligned}
\left(\frac{d}{d s}-\tilde{\Delta}\right) f^{2} \tilde{\rho}(\tilde{x}, s) \leq & -2 f^{2} \tilde{\rho}-2 \frac{\tilde{\rho}}{\tilde{v}} \nabla \tilde{v} \cdot \nabla f^{2}+f^{2}\left(\frac{d}{d s}-\tilde{\Delta}\right) \tilde{\rho} \\
& -2 \nabla \tilde{\rho} \cdot \nabla f^{2} .
\end{aligned}
$$


Note that since $\nabla\left(f^{2} \tilde{\rho}\right)=\tilde{\rho} \nabla f^{2}+f^{2} \nabla \tilde{\rho}$, we can write

$$
\begin{aligned}
-2 \frac{\tilde{\rho}}{\tilde{v}} \nabla \tilde{v} \cdot \nabla f^{2}-2 \nabla \tilde{\rho} \cdot \nabla f^{2}= & -2\left(\frac{\nabla \tilde{v}}{\tilde{v}}+\frac{\nabla \tilde{\rho}}{\tilde{\rho}}\right) \nabla\left(f^{2} \tilde{\rho}\right)+2 \frac{f^{2}}{\tilde{v}} \nabla \tilde{\rho} \cdot \nabla \tilde{v} \\
& +2 \frac{f^{2}}{\tilde{\rho}}|\nabla \tilde{\rho}|^{2}
\end{aligned}
$$

so that we end up with

$$
\begin{aligned}
\left(\frac{d}{d s}-\tilde{\Delta}\right) f^{2} \tilde{\rho}(\tilde{x}, s) \leq & -2 f^{2} \tilde{\rho}+f^{2}\left(\frac{d}{d s}-\tilde{\Delta}\right) \tilde{\rho}+2 \frac{f^{2}}{\tilde{\rho}}|\nabla \tilde{\rho}|^{2} \\
& -2\left(\frac{\nabla \tilde{v}}{\tilde{v}}+\frac{\nabla \tilde{\rho}}{\tilde{\rho}}\right) \nabla\left(f^{2} \tilde{\rho}\right)+2 \frac{f^{2}}{\tilde{v}} \nabla \tilde{\rho} \cdot \nabla \tilde{v}
\end{aligned}
$$

Proof of Main Theorem. When we set $h(s)=(1+s)^{\gamma}$ for some positive $\gamma$, then we have that:

$$
h^{\prime}(s)=\frac{\gamma}{1+s} h(s)
$$

thus giving us the following estimate for the evolution of $\tilde{\rho}$ via Proposition 4.1

$$
\begin{aligned}
\left(\frac{d}{d s}-\tilde{\Delta}\right) \tilde{\rho}(\tilde{x}, s) \leq & \epsilon \frac{\tilde{\rho}}{\log \tilde{\eta}_{1}}\left(\frac{d}{d s}-\tilde{\Delta}\right) \tilde{\eta}_{1}+\epsilon\left(1-\frac{\epsilon}{2}\right) \frac{\left|\nabla \tilde{\eta}_{1}\right|^{2}}{\tilde{\eta}_{1}^{2} \log ^{2} \tilde{\eta}_{1}} \tilde{\rho} \\
& -p \frac{\tilde{\rho}}{\tilde{\eta}_{2}}\left(\frac{d}{d s}-\tilde{\Delta}\right) \tilde{\eta}_{2}+p(p-1) \frac{\left|\nabla \tilde{\eta}_{2}\right|^{2}}{\tilde{\eta}_{2}^{2}} \tilde{\rho} \\
& +\frac{\gamma}{1+s} \tilde{\rho} .
\end{aligned}
$$

Now define $\tilde{\eta}_{1}$ and $\tilde{\eta}_{2}$ as

$$
\tilde{\eta}_{1}=e+\alpha|\tilde{x}|^{2} \quad \text { and } \quad \tilde{\eta}_{2}=1+\beta|\tilde{x}|^{2}-\beta \tilde{u}^{2}
$$

for some positive constants $\alpha$ and $\beta$ to be determined later. We have that since both $|x|^{2}$ and $u^{2}$ are of "degree" 2 , together with the fact that

$$
\left(\frac{d}{d t}-\Delta\right)|x|^{2}=-2 n \quad \text { and } \quad\left(\frac{d}{d t}-\Delta\right) u^{2}=-2|\nabla u|^{2}
$$

the heat operator of $\tilde{\eta}_{1}$ is given by:

$$
\left(\frac{d}{d s}-\tilde{\Delta}\right) \tilde{\eta}_{1}=-2 \alpha\left(|\tilde{x}|^{2}+n\right)
$$


and that of $\tilde{\eta}_{2}$ by:

$$
\left(\frac{d}{d s}-\tilde{\Delta}\right) \tilde{\eta}_{2}=-2 \beta\left(|\tilde{x}|^{2}+n\right)+2 \beta\left(|\nabla \tilde{u}|^{2}+\tilde{u}^{2}\right) .
$$

This then implies the following estimate for $0<p<1,0<\epsilon<\delta$ and $s>0$ :

$$
\begin{aligned}
\left(\frac{d}{d s}-\tilde{\Delta}\right) \tilde{\rho} \leq & 2\left(p \beta-\frac{\epsilon \alpha}{\log \tilde{\eta}_{1}}\right)\left(|\tilde{x}|^{2}+n\right) \tilde{\rho} \\
& +\frac{1}{2} \frac{\left|\nabla \tilde{\eta}_{1}\right|^{2}}{\tilde{\eta}_{1}^{2} \log ^{2} \tilde{\eta}_{1}} \tilde{\rho}+\frac{\gamma}{1+s} \tilde{\rho} \\
\leq & \frac{1}{2} \frac{\left|\nabla \tilde{\eta}_{1}\right|^{2}}{\tilde{\eta}_{1}^{2} \log ^{2} \tilde{\eta}_{1}} \tilde{\rho}+\gamma \tilde{\rho}
\end{aligned}
$$

where we have chosen $\beta$ such that $\beta \leq \epsilon \alpha / p \log \eta_{1}$, so that we can drop first term in the above inequality and that $\epsilon-\epsilon^{2} / 2 \leq 1 / 2$ for $\epsilon>0$.

Moreover we obtain from the estimates

$$
\left|\nabla \tilde{\eta}_{1}\right|^{2} \leq 4 \alpha \tilde{\eta}_{1} \quad \text { and } \quad\left|\nabla \tilde{\eta}_{2}\right|^{2} \leq 4 \beta \tilde{\eta}_{2}
$$

the following estimates:

$$
\left(\frac{d}{d s}-\tilde{\Delta}\right) \tilde{\rho} \leq(2 \alpha+\gamma) \tilde{\rho}
$$

and

$$
\begin{aligned}
|\nabla \tilde{\rho}| & \leq\left(\epsilon \frac{\left|\nabla \tilde{\eta}_{1}\right|}{\tilde{\eta}_{1} \log \tilde{\eta}_{1}}+p \frac{\left|\nabla \tilde{\eta}_{2}\right|}{\tilde{\eta}_{2}}\right) \tilde{\rho} \\
& \leq 2(\epsilon \sqrt{\alpha}+p \sqrt{\beta}) \tilde{\rho} \leq 2 \sqrt{\epsilon \alpha}(\sqrt{\epsilon}+\sqrt{p}) \tilde{\rho}
\end{aligned}
$$

which then gives

$$
2 \frac{f^{2}}{\tilde{\rho}}|\nabla \tilde{\rho}|^{2} \leq 8 \epsilon \alpha(\sqrt{\epsilon}+\sqrt{p})^{2} f^{2} \tilde{\rho}
$$

and together with the estimate $|\nabla \tilde{v}| / \tilde{v} \leq|\tilde{A}| \tilde{v} \leq c_{1} c(0)$ also gives:

$$
2 \frac{f^{2}}{\tilde{v}} \nabla \tilde{\rho} \cdot \nabla \tilde{v} \leq c\left(c_{1}, c(0), n\right) \sqrt{\epsilon \alpha}(\sqrt{\epsilon}+\sqrt{p}) f^{2} \tilde{\rho} .
$$


Thus we finally have after substituting the above estimate into (4.2):

$$
\begin{aligned}
\left(\frac{d}{d s}-\tilde{\Delta}\right) f^{2} \tilde{\rho} \leq & \vec{a} \cdot \nabla\left(f^{2} \tilde{\rho}\right)+(2 \alpha+\gamma+c \sqrt{\epsilon \alpha}(\sqrt{\epsilon}+\sqrt{p}) \\
& \left.+8 \epsilon \alpha(\sqrt{\epsilon}+\sqrt{p})^{2}-2\right) f^{2} \tilde{\rho}
\end{aligned}
$$

where

$$
\vec{a}=-2\left(\frac{\nabla \tilde{v}}{\tilde{v}}+\frac{\nabla \tilde{\rho}}{\tilde{\rho}}\right) .
$$

Choosing $\alpha, \beta$ and $\gamma$ suitably small depending on $\epsilon, p$ and $c$, we see that:

$$
\left(\frac{d}{d s}-\tilde{\Delta}\right) f^{2} \tilde{\rho} \leq \vec{a} \cdot \nabla\left(f^{2} \tilde{\rho}\right),
$$

for all $s>0$. Lemma 6.1 ensures that $f^{2} \tilde{\rho}$ vanishes at infinity which enables us to apply the parabolic maximum principle to conclude that $f^{2} \tilde{\rho}$ is uniformly bounded by its initial data.

Finally we use the result of Stavrou [3] to conclude uniform convergence to self-similar solutions, since our assumption is stronger than his.

\section{Acknowledgment}

This research was supported by the IMPRS at the Albert Einstein Institute, Potsdam-Golm and the SFB 647: Space Time Matter.

\section{References}

[1] K. Ecker and G. Huisken, Mean curvature evolution of entire graphs, Ann. Math. 130(3) (1989), 453-471.

[2] G. Huisken, Asymptotic behavior for singularities of the mean curvature flow, J. Differential Geom. 31 (1990), 285-299.

[3] N. Stavrou, Selfsimilar solutions to the mean curvature flow, J. Reine Angew. Math. 499 (1998), 189-198.

Freie UNIVERsitätT BerLin

FACHBEREICH MATHEMATIK UND INFORMATIK

Arnimallee 3

14195 Berlin, Germany

E-mail address: kashif.rasul@fu-berlin.de

Received August 17, 2010 